\title{
An Observational Study of Outcome in Blunt Abdominal Trauma Managed Conservatively.
}

\author{
Shshank P Jain, Abha G Gune, G.S Narshetty
}

\begin{abstract}
Trauma remains the most common cause of death for all individuals between the ages of 1 and 44 years and is the third most common cause of death regardless of age according to western data. The care of the trauma patient is demanding and requires speed and efficiency. Evaluating patients who have sustained blunt abdominal trauma remains one of the most challenging and resource-intensive aspects of acute trauma care. Our study is based on evaluation of 100 patients admitted in emergency department of a tertiary care centre with features of blunt abdominal trauma who were managed conservatively after thorough evaluation of patient and excluding any immediate operative intervention. This study establishes various outcomes of patients being managed conservatively after a blunt abdominal trauma .
\end{abstract}

\section{Introduction}

Trauma-care systems in India are at a nascent stage of development. Industrialized cities, rural towns and villages coexist, with variety of health care facilities and almost complete lack of organized trauma care. There is gross disparity between trauma services available in various parts of the country. Rural India has inefficient services for trauma care, due to the varied topography, financial constraints and lack of appropriate health infrastructure. (1)

Out of the many categories for trauma, blunt trauma to abdomen, forms one of the major categories of those patients, in whom, death can be prevented with timely intervention. (2).

However most avoidable fatalities occur as a result of failed resuscitation and failure to recognise surgically correctable injuries.(3)

Physical examination findings are notoriously unreliable. One reason is that mechanisms of injury often result in other associated injuries that may divert the physician's attention from potentially life-threatening intra-abdominal pathology. Other common reasons are an altered mental state and drug and alcohol intoxication. Coordinating trauma resuscitation, demands a thorough understanding of the pathophysiology of trauma and shock, excellent clinical and diagnostic acumen, skill with complex procedures, compassion, and the ability to think rationally in a chaotic milieu.(2)

When the diagnosis is in doubt and clinical judgment suggest surgery, going ahead with exploration provides definitive treatment as well as a diagnosis, although a risk of negative exploration unless justified can increase the fatality.(4)

Blunt abdominal trauma usually results from motor vehicle collisions (MVCs), assaults, recreational accidents, or falls. The most commonly injured organs are the spleen, liver, retroperitoneum, small bowel, kidneys, bladder, colorectum, diaphragm, and pancreas. Men tend to be affected slightly more often than women.(2)

Our study highlights the various outcomes of patients who had been admitted with blunt abdominal trauma and were managed conservatively.

\section{Aims And Objectives}

1) To demonstrate the outcome of managing patients of blunt abdominal trauma based on age, sex of patient , abdominal organ involved and mechanism of injury

2) To demonstrate relation between co-morbidities, distracting injuries, age group ,nutrition of patient and complications with hospital stay of patients with blunt abdominal trauma .

\section{Materials And Method}

This study was performed at Mahatma Gandhi Mission hospital, Kamothe, New Mumbai, Maharashtra, for a period of 2 years from May 2012 to April 2014 after approval by the institutional ethical committee done in September 2012. It was a prospective, analytical study which included 100 patients of trauma that were admitted from the emergency department of the hospital. The study included all the cases admitted with blunt trauma to the abdomen.

All the patients of blunt abdominal trauma, after initial resuscitation were evaluated clinically with respect to the symptoms and signs in the emergency room itself. This was aided with Focussed abdominal sonar 
for trauma (FAST) and abdominal radiograph performed in the emergency department itself by a qualified radiologist. Also, the patients were subjected to Computerised tomography (CT scan).

Based on the clinical and radiological findings, patients were either managed conservatively or operatively. Patients who were hemodynamically unstable even after resuscitation and those who showed gas under diaphragm on erect/lateral abdominal radiograph, as a sign of perforation were immediately taken up for surgery and were thus excluded from our study group. Our study group involved patients who were initially resuscitated and responded to this line of management and hence conservatively managed. Each patient was granted a risk score that showed whether the patient could be managed conservatively or would require surgical intervention.

Our risk factor scoring was based on 20 clinical parameters which involved vitals like pulse ,blood pressure ,age of patient ,mechanism of injury ,respiratory rate ,saturation of oxygen as per pulse oximeter ,comorbid conditions of patient (eg .Ischemic heart diseases ,diabetes) ,built and nutrition ,distracting injuries, imprint abrasion , pallor ,abdominal tenderness, guarding ,rigidity, coastal tenderness , pelvic tenderness ,flank pain ,abdominal distention, haematuria and usg score for hemoperitoneum. Using these parameters our patients were grouped into three categories:

1. Low risk: patients who did not require any surgical intervention and could be managed in the wards.

2. Moderate risk: These patients were managed in intensive care unit. These were the ones who did not need a surgical intervention but required intensive monitoring and management. These were potential candidates for operative intervention if chances of the outcome being better than the conservative approach were justified.

3. High risk: These patients were poor candidates for conservative management but had responded to initial treatment . These patients at some point would require surgery.

All the patients were assessed on guidelines as per trauma protocols and each patient is evaluated by the researcher. All patients have been evaluated radiologically by use of Ultrasonography and X-rays. CT scan use was strictly reserved for patients who lie in the category of moderate to high risk category as given by the researcher and at no point was the health and treatment of patient compromised in name of research.

Patients who were assigned moderate to high risk by scoring in emergency were all admitted to Surgical Intensive Care Unit (SICU) where all the parameters were evaluated every half hour for first 24 hrs and then every hour for the rest of the patient's stay. Ultrasound of the abdomen was repeated every 4hours for patients who were stated high risk by our scoring and every 6hours for patients who were at moderate risk. Patients who initially responded to resuscitation but later were unable to maintain their vitals or ultrasound showed any positive findings which prompted surgery were then declared as patients unfit for conservative therapy and delayed laparotomy was undertaken.

The inclusion and exclusion criterion for our study was as follows:

Inclusion Criteria:1)Age 18yrs and above. 2)History of blunt trauma to abdomen $<24 \mathrm{hrs}$

Exclusion Criteria:1)Penetrating injuries .2)Head injury component GCS <14 . 3)Age <18.

4)Pregnant females

\section{Results}

Table 1: Distribution of Data by Age-Group

\begin{tabular}{|l|l|l|}
\hline AGE GROUP & NUMBER OF CASES & PERCENTAGE \\
\hline $18-30$ & 40 & 40.0 \\
\hline $31-50$ & 41 & 41.0 \\
\hline$>50$ & 19 & 19.0 \\
\hline TOTAL & 100 & 100 \\
\hline
\end{tabular}

The above data shows age distribution among our trauma patients. Our study shows 41 percent of adults in age group of 31-50 and a close 40 percent of young patients in age range of 18-30. Adults above age 50 forms a small group of patients ie. 19 percent.

Table 2 : Distribution of Data by Sex

\begin{tabular}{|l|l|l|}
\hline SEX & NUMBER OF CASES & PERCENTAGE \\
\hline MALE & 85 & 85.0 \\
\hline FEMALE & 15 & 15.0 \\
\hline TOTAL & 100 & 100 \\
\hline
\end{tabular}

The above data shows that maximum number of patients belongs to male gender forming 85 percent of our study group and females form a mere 15 percent. 
Table 3: Distribution of Data by Etiology of Injuries

\begin{tabular}{|l|l|l|}
\hline Nature Of Injury & Number Of Cases & Percentage \\
\hline Road Traffic Accidents & 63 & 63.0 \\
\hline Fall & 21 & 21.0 \\
\hline Fall Of Heavy Object & 7 & 7.0 \\
\hline Assault & 9 & 9.0 \\
\hline Total & 100 & 100 \\
\hline
\end{tabular}

Road traffic accidents form a major etiology of blunt abdominal trauma with $63 \%$ and a fall from height following a close second with $21 \%$.

Table 4: Distribution of Data by Signs

\begin{tabular}{|l|l|l|}
\hline Signs & Number Of Cases & Percentage \\
\hline Abdominal Tenderness & 96 & 96 \\
\hline Guarding & 62 & 62 \\
\hline Rigidity & 10 & 10 \\
\hline Coastal Margin Tenderness & 53 & 53 \\
\hline Pelvic Tenderness & 25 & 25 \\
\hline Flank Pain & 29 & 29 \\
\hline Abdominal Distension & 12 & 12 \\
\hline Haematuria & 19 & 19 \\
\hline
\end{tabular}

Abdominal tenderness forms a major presentation when it comes to blunt abdominal trauma as it is present in almost all patients occupying $96 \%$ followed closely by guarding and coastal margin tenderness at $62 \%$ and $53 \%$ respectively.

Table 5: Distribution of Data by Duration of Hospital Stay

\begin{tabular}{|l|l|l|}
\hline Duration Of Stay (Days) & Number Of Cases & Percentage \\
\hline $1-5$ & 51 & 51.0 \\
\hline $6-10$ & 14 & 14.0 \\
\hline $11-15$ & 8 & 8.0 \\
\hline $16-20$ & 5 & 5.0 \\
\hline$>20$ & 22 & 22.0 \\
\hline Total & 100 & 100 \\
\hline
\end{tabular}

Hospital duration stay depends upon many factors; most of the conservatively managed patients have a maximum of 5 days stay. Hospital stay increases with presence of co-morbid conditions, other concurrent injuries, old age, mechanism of injury and many other factors. Hospital stay reflects the nature of injury and is directly proportional to impact the injury has on patient outcome.

Table 6: Distribution of Data by Conservative Management

\begin{tabular}{|l|l|l|}
\hline Consrvative Management & Number Of Cases & Percentage \\
\hline Yes & 83 & 83.0 \\
\hline No & 17 & 17.0 \\
\hline Total & 100 & 100 \\
\hline
\end{tabular}

Table 7 : Distribution of Data by Delayed Laparotomy Management

\begin{tabular}{|l|l|l|}
\hline Delayed Laparotomy Management & Number of Cases & Percentage \\
\hline Yes & 17 & 17.0 \\
\hline No & 83 & 83.0 \\
\hline Total & 100 & 100 \\
\hline
\end{tabular}

The above two data shows that among our study group of 100 patients who were managed conservatively only 17 percent underwent delayed laparotomy while maximum number of patients ie.83 percent were successfully managed conservatively.

Table 8 : Distribution of Data by Management

\begin{tabular}{|l|l|l|}
\hline Treatment & Number Of Cases & Percentage \\
\hline Consrvative Management & 83 & 83.0 \\
\hline Delayed Laparotomy Management & 17 & 17.0 \\
\hline Total & 100 & 100 \\
\hline
\end{tabular}


Table 9: Distribution of Data by Complications

\begin{tabular}{|l|l|l|}
\hline Complications & Number of Cases & Percentage \\
\hline None & 57 & 57.0 \\
\hline Sepsis & 9 & 9.0 \\
\hline Mi & 3 & 3.0 \\
\hline Wound Infection And Anastomsis & 1 & 1.0 \\
\hline Intestinal Obstruction & 4 & 4.0 \\
\hline Wound Infection & 5 & 5.0 \\
\hline Pnemonia & 6 & 6.0 \\
\hline Hypertensive Crisis & 4 & 4.0 \\
\hline Ketoacidosis & 3 & 3.0 \\
\hline Pleural Effusion & 2 & 2.0 \\
\hline Pulmonary Embolism & 1 & 1.0 \\
\hline Pnemonia And Dvt & 1 & 1.0 \\
\hline Ards & 2 & 2.0 \\
\hline Mi With Ards & 2 & 2.0 \\
\hline Total & 100 & 100 \\
\hline
\end{tabular}

TABLE 9 showing complicated and uncomplicated cases in operative and conservative management. Most of the patients managed conservatively did not have any complications. Most common complication was sepsis and pneumonia ie. $9 \%$ and $6 \%$ respectively. Out of the 43 patients who had complications $25.6 \%$ cases were post operative complication forming $64.7 \%$ of total number of operative cases. While $74.4 \%$ of cases under complication belonged to conservative management, which formed $38.5 \%$ of the total cases managed conservatively.

Table 10 : Distribution of Data by Diagnosis (Organ Involvement)

\begin{tabular}{|l|l|l|}
\hline Diagnosis & Number Of Cases & Percentage \\
\hline Liver Laceration & 12 & 12.0 \\
\hline Splenic Laceration & 9 & 9.0 \\
\hline Meseteric Injury & 39 & 39.0 \\
\hline Large Bowel Injury & 1 & 1.0 \\
\hline Small Bowel Injury & 2 & 2.0 \\
\hline Renal Laceration & 3 & 3.0 \\
\hline Liver Contusion & 13 & 13.0 \\
\hline Bladder Contusion & 4 & 4.0 \\
\hline Splenic Contusion & 10 & 10.0 \\
\hline Renal Contusion & 6 & 6.0 \\
\hline Pancreatic Injury & 1 & 1.0 \\
\hline Total & 100 & 100 \\
\hline
\end{tabular}

In the above data its shows most commonly injured organ was mesentery (39\%) followed closely by liver (25 $\%)$ and spleen (19\%) involvement. Least injured organ was pancreas which formed only $1 \%$.

Table 11 : Distribution of Data by Mortality

\begin{tabular}{|l|l|l|}
\hline Mortality & Number Of Cases & Percentage \\
\hline None & 97 & 97.0 \\
\hline Yes & 3 & 3.0 \\
\hline Total & 100 & 100 \\
\hline
\end{tabular}


An Observational Study of Outcome in Blunt Abdominal Trauma Managed Conservatively.

Table 12: Cross Tabulation of Data of Distract Injuries by Duration of Hospital Stay

1-5 DAYS $=1,6-10=2,11-15=3,16-20=4,>20=5$ * LOW RISK=1, MODERATE RISK=2, HIGH RISK=3 Crosstabulation

\begin{tabular}{|c|c|c|c|c|c|c|}
\hline & & & LOW RISF & $\begin{array}{l}\text { MODERA } \\
\text { H RISK= }\end{array}$ & RISK=2, & \\
\hline & & & 1.00 & 2.00 & 3.00 & Total \\
\hline 1-5 DAYS $=1$ & 1.00 & Count & 47 & 3 & 1 & 51 \\
\hline $\begin{array}{l}6-10=2,11-15=3, \\
16-20=4,>20=5\end{array}$ & & $\begin{array}{l}\% w \text { ithin } 1-5 \text { DAYS }=1 \text {, } \\
6-10=2,11-15=3, \\
16-20=4,>20=5\end{array}$ & $92.2 \%$ & $5.9 \%$ & $2.0 \%$ & $100.0 \%$ \\
\hline & & $\%$ of Total & $47.0 \%$ & $3.0 \%$ & $1.0 \%$ & $51.0 \%$ \\
\hline & 2.00 & Count & 9 & 5 & & 14 \\
\hline & & $\begin{array}{l}\% \text { within } 1-5 \text { DAYS }=1 \text {, } \\
6-10=2,11-15=3, \\
16-20=4,>20=5\end{array}$ & $64.3 \%$ & $35.7 \%$ & & $100.0 \%$ \\
\hline & & $\%$ of Total & $9.0 \%$ & $5.0 \%$ & & $14.0 \%$ \\
\hline & 3.00 & Count & 4 & 2 & 2 & 8 \\
\hline & & $\begin{array}{l}\% w \text { ithin } 1-5 \text { DAYS }=1, \\
6-10=2,11-15=3 \\
16-20=4,>20=5\end{array}$ & $50.0 \%$ & $25.0 \%$ & $25.0 \%$ & $100.0 \%$ \\
\hline & & $\%$ of Total & $4.0 \%$ & $2.0 \%$ & $2.0 \%$ & $8.0 \%$ \\
\hline & 4.00 & Count & & 4 & 1 & 5 \\
\hline & & $\begin{array}{l}\% w \text { ithin } 1-5 \text { DAYS }=1, \\
6-10=2,11-15=3, \\
16-20=4,>20=5\end{array}$ & & $80.0 \%$ & $20.0 \%$ & $100.0 \%$ \\
\hline & & $\%$ of Total & & $4.0 \%$ & $1.0 \%$ & $5.0 \%$ \\
\hline & 5.00 & Count & 7 & 6 & 9 & 22 \\
\hline & & $\begin{array}{l}\% \text { w ithin } 1-5 \text { DAYS }=1, \\
6-10=2,11-15=3, \\
16-20=4,>20=5\end{array}$ & $31.8 \%$ & $27.3 \%$ & $40.9 \%$ & $100.0 \%$ \\
\hline & & $\%$ of Total & $7.0 \%$ & $6.0 \%$ & $9.0 \%$ & $22.0 \%$ \\
\hline Total & & Count & 67 & 20 & 13 & 100 \\
\hline & & $\begin{array}{l}\% \text { within } 1-5 \text { DAYS }=1 \text {, } \\
6-10=2,11-15=3, \\
16-20=4,>20=5\end{array}$ & $67.0 \%$ & $20.0 \%$ & $13.0 \%$ & $100.0 \%$ \\
\hline & & $\%$ of Total & $67.0 \%$ & $20.0 \%$ & $13.0 \%$ & $100.0 \%$ \\
\hline
\end{tabular}

Chi-Square Tests

\begin{tabular}{|c|c|c|c|}
\hline & Value & df & $\begin{array}{l}\text { As ymp. Sig. } \\
\text { (2-sided) }\end{array}$ \\
\hline Pearson Chi-Square & 49.942 & 8 & .000 \\
\hline Likelihood Ratio & 50.946 & 8 & .000 \\
\hline $\begin{array}{l}\text { Linear-by-Linear } \\
\text { As sociation }\end{array}$ & 35.672 & 1 & .001 \\
\hline N of Valid Cases & 100 & & \\
\hline
\end{tabular}

V. Correlation Analysis

\begin{tabular}{|l|r|r|}
\hline & Value & Approx. Sig. \\
\hline Pearson's R & .875 & .000 \\
Spearman Correlation & .852 & .000 \\
\hline
\end{tabular}

$* \mathbf{P}<0.05=$ Significant

$* \mathbf{P}>0.05=$ Not Significant

*Moderate Risk and High Risk Shows Highly and Greater Significant

*Moderate Risk and High Risk Shows Duration of Hospital Stay in Days Increases

* Pearson Chi-Square Test Shows Statistical Difference between Groups, P Value and Significance

The above data concludes that distracting injuries that is injuries other than injury to abdomen increases the hospital stay and hence increases the morbidity, the health care cost and affects the final outcome. 
An Observational Study of Outcome in Blunt Abdominal Trauma Managed Conservatively.

Table 13: Cross Tabulation of Data of Comorbidities by Duration of Hospital Stay

1-5 DAYS $=1,6-10=2,11-15=3,16-20=4,>20=5$ * LOW RISK $=1$, MODERATE RISK $=2$, HIGH RISK=3 Crosstabulation

\begin{tabular}{|c|c|c|c|c|c|c|}
\hline & & & LOW RISH & $\begin{array}{l}\text { MODERA } \\
\mathrm{H} \text { RISK= }\end{array}$ & $\mathrm{RISK}=2$, & \\
\hline & & & 1.00 & 2.00 & 3.00 & Total \\
\hline 1-5 DAYS=1, & 1.00 & Count & 42 & 8 & 1 & 51 \\
\hline $\begin{array}{l}6-10=2,11-15=3 \\
16-20=4,>20=5\end{array}$ & & $\begin{array}{l}\% \text { w ithin } 1-5 \text { DAYS }=1 \text {, } \\
6-10=2,11-15=3 \\
16-20=4,>20=5\end{array}$ & $82.4 \%$ & $15.7 \%$ & $2.0 \%$ & $100.0 \%$ \\
\hline & & $\%$ of Total & $42.0 \%$ & $8.0 \%$ & $1.0 \%$ & $51.0 \%$ \\
\hline & 2.00 & Count & 9 & 3 & 2 & 14 \\
\hline & & $\begin{array}{l}\% \text { w ithin } 1-5 \text { DAYS }=1 \text {, } \\
6-10=2,11-15=3 \\
16-20=4,>20=5\end{array}$ & $64.3 \%$ & $21.4 \%$ & $14.3 \%$ & $100.0 \%$ \\
\hline & & $\%$ of Total & $9.0 \%$ & $3.0 \%$ & $2.0 \%$ & $14.0 \%$ \\
\hline & 3.00 & Count & 3 & 3 & 2 & 8 \\
\hline & & $\begin{array}{l}\% \text { w ithin } 1-5 \text { DAYS }=1, \\
6-10=2,11-15=3 \\
16-20=4,>20=5\end{array}$ & $37.5 \%$ & $37.5 \%$ & $25.0 \%$ & $100.0 \%$ \\
\hline & & $\%$ of Total & $3.0 \%$ & $3.0 \%$ & $2.0 \%$ & $8.0 \%$ \\
\hline & 4.00 & Count & 3 & 2 & & 5 \\
\hline & & $\begin{array}{l}\% w \text { ithin } 1-5 \text { DAYS }=1 \\
6-10=2,11-15=3 \\
16-20=4,>20=5\end{array}$ & $60.0 \%$ & $40.0 \%$ & & $100.0 \%$ \\
\hline & & $\%$ of Total & $3.0 \%$ & $2.0 \%$ & & $5.0 \%$ \\
\hline & 5.00 & Count & 11 & 3 & 8 & 22 \\
\hline & & $\begin{array}{l}\% \text { w ithin } 1-5 \text { DAYS }=1 \text {, } \\
6-10=2,11-15=3 \\
16-20=4,>20=5\end{array}$ & $50.0 \%$ & $13.6 \%$ & $36.4 \%$ & $100.0 \%$ \\
\hline & & $\%$ of Total & $11.0 \%$ & $3.0 \%$ & $8.0 \%$ & $22.0 \%$ \\
\hline Total & & Count & 68 & 19 & 13 & 100 \\
\hline & & $\begin{array}{l}\% \text { w ithin } 1-5 \text { DAYS }=1 \text {, } \\
6-10=2,11-15=3 \\
16-20=4,>20=5\end{array}$ & $68.0 \%$ & $19.0 \%$ & $13.0 \%$ & $100.0 \%$ \\
\hline & & $\%$ of Total & $68.0 \%$ & $19.0 \%$ & $13.0 \%$ & $100.0 \%$ \\
\hline
\end{tabular}

Chi-Square Tests

\begin{tabular}{|c|c|c|c|}
\hline & Value & df & $\begin{array}{l}\text { As ymp. Sig. } \\
\text { (2-sided) }\end{array}$ \\
\hline Pearson Chi-Square & 22.608 & 8 & .004 \\
\hline Likelihood Ratio & 22.379 & 8 & .004 \\
\hline $\begin{array}{l}\text { Linear-by-Linear } \\
\text { Association }\end{array}$ & 13.649 & 1 & .000 \\
\hline$N$ of $V$ alid Cases & 100 & & \\
\hline
\end{tabular}

Sym metric Measures

\begin{tabular}{|l|r|r|}
\hline & Value & Approx. Sig. \\
\hline Pearson's R & .847 & .000 \\
Spearman Correlation & .862 & .001 \\
\hline
\end{tabular}

$* \mathbf{P}<\mathbf{0 . 0 5}=$ Significant

$* \mathbf{P}>0.05=$ Not Significant

*Moderate Risk And High Risk Shows Highly And Greater Significance

*Moderate Risk And High Risk Shows Duration Of Hospital Stay In Days Increases

* Pearson Chi-Square Test Shows Statistical Difference Between Groups, P Value And Significance

The above data shows that patients with co-morbid conditions like medical history of diabetes, ischemic heart diseases, hypertension increases the morbidity and mortality .It finally affects the outcome irrespective of intervention taken. 
An Observational Study of Outcome in Blunt Abdominal Trauma Managed Conservatively.

Table 14: Cross Tabulation of Data of Age in Range by Duration of Hospital Stay

1-5 DAYS=1, 6-10=2, 11-15=3, 16-20=4, >20=5 * LOW RISK=1, MODERATE RISK=2, HIGH RISK=3 Crosstabulation

\begin{tabular}{|c|c|c|c|c|c|c|}
\hline & & & LOW RIS & $\begin{array}{l}\text { MODER } \\
\text { H RISK }\end{array}$ & ISK=2, & \\
\hline & & & 1.00 & 2.00 & 3.00 & Total \\
\hline 1-5DAYS $=1$, & 1.00 & Count & 22 & 18 & 11 & 51 \\
\hline $\begin{array}{l}6-10=2,11-15=3, \\
16-20=4,>20=5\end{array}$ & & $\begin{array}{l}\% \text { w ithin } 1-5 \text { DAYS=1, } \\
6-10=2,11-15=3, \\
16-20=4,>20=5\end{array}$ & $43.1 \%$ & $35.3 \%$ & $21.6 \%$ & $100.0 \%$ \\
\hline & & $\%$ of Total & $22.0 \%$ & $18.0 \%$ & $11.0 \%$ & $51.0 \%$ \\
\hline & 2.00 & Count & 8 & 4 & 2 & 14 \\
\hline & & $\begin{array}{l}\% \text { w ithin } 1-5 \text { DAYS }=1 \text {, } \\
6-10=2,11-15=3, \\
16-20=4,>20=5\end{array}$ & $57.1 \%$ & $28.6 \%$ & $14.3 \%$ & $100.0 \%$ \\
\hline & & $\%$ of Total & $8.0 \%$ & $4.0 \%$ & $2.0 \%$ & $14.0 \%$ \\
\hline & 3.00 & Count & 1 & 6 & 1 & 8 \\
\hline & & $\begin{array}{l}\% \text { w ithin } 1-5 \text { DAYS }=1 \text {, } \\
6-10=2,11-15=3, \\
16-20=4,>20=5\end{array}$ & $12.5 \%$ & $75.0 \%$ & $12.5 \%$ & $100.0 \%$ \\
\hline & & $\%$ of Total & $1.0 \%$ & $6.0 \%$ & $1.0 \%$ & $8.0 \%$ \\
\hline & 4.00 & Count & 1 & 3 & 1 & 5 \\
\hline & & $\begin{array}{l}\% \text { w ithin } 1-5 \text { DAYS=1, } \\
6-10=2,11-15=3, \\
16-20=4,>20=5\end{array}$ & $20.0 \%$ & $60.0 \%$ & $20.0 \%$ & $100.0 \%$ \\
\hline & & $\%$ of Total & $1.0 \%$ & $3.0 \%$ & $1.0 \%$ & $5.0 \%$ \\
\hline & 5.00 & Count & 7 & 10 & 5 & 22 \\
\hline & & $\begin{array}{l}\% \text { w ithin } 1-5 \text { DAYS=1, } \\
6-10=2,11-15=3, \\
16-20=4,>20=5\end{array}$ & $31.8 \%$ & $45.5 \%$ & $22.7 \%$ & $100.0 \%$ \\
\hline & & $\%$ of Total & $7.0 \%$ & $10.0 \%$ & $5.0 \%$ & $22.0 \%$ \\
\hline Total & & Count & 39 & 41 & 20 & 100 \\
\hline & & $\begin{array}{l}\% \text { w ithin } 1-5 \text { DAYS }=1 \text {, } \\
6-10=2,11-15=3, \\
16-20=4,>20=5\end{array}$ & $39.0 \%$ & $41.0 \%$ & $20.0 \%$ & $100.0 \%$ \\
\hline & & $\%$ of Total & $39.0 \%$ & $41.0 \%$ & $20.0 \%$ & $100.0 \%$ \\
\hline
\end{tabular}

Chi-Square Tests

\begin{tabular}{|c|c|c|c|}
\hline & Value & df & $\begin{array}{c}\text { As ymp. Sig. } \\
\text { (2-sided) }\end{array}$ \\
\hline Pearson Chi-Square & 27.933 & 8 & .004 \\
\hline Likelihood Ratio & 28.051 & 8 & .002 \\
\hline $\begin{array}{l}\text { Linear-by-Linear } \\
\text { Association }\end{array}$ & 24.853 & 1 & .003 \\
\hline$N$ of $V$ alid Cases & 100 & & \\
\hline
\end{tabular}

Sym metric Measures

\begin{tabular}{|l|r|r|}
\hline & \multicolumn{1}{|c|}{ Value } & Approx. Sig. \\
\hline Pearson's R & .932 & .003 \\
Spearman Correlation & .822 & .004 \\
\hline
\end{tabular}

$* \mathbf{P}<0.05=$ Significant

*P $>$ 0.05= Not Significant

*Moderate Risk and High Risk Shows Highly and Greater Significant

*Moderate Risk and High Risk Shows Duration of Hospital Stay in Days Increases

* Pearson Chi-Square Test Shows Statistical Difference between Groups, $\mathbf{P}$ Value and Significance

The above study shows that younger age group responds better to treatment modalities with positive outcomes and minimum complications and hence an early discharge from hospital compared to older age group which are bound for poor response to interventions weather conservative or operative. 
An Observational Study of Outcome in Blunt Abdominal Trauma Managed Conservatively.

Table 15: Cross Tabulation of Data of Mechanism of Injury by Duration of Hospital Stay

1-5 DAYS $=1,6-10=2,11-15=3,16-20=4,>20=5$ * LOW RISK $=1$, MODERATE RISK=2, HIGH RISK=3 Crosstabulation

\begin{tabular}{|c|c|c|c|c|c|c|}
\hline & & & \multicolumn{3}{|c|}{$\begin{array}{c}\text { LOW RISK }=1 \text {, MODERATE RISK=2, } \\
\text { HIGH RISK }=3\end{array}$} & \multirow[b]{2}{*}{ Total } \\
\hline & & & 1.00 & 2.00 & 3.00 & \\
\hline \multirow{15}{*}{$\begin{array}{l}1-5 \text { DAY }=1, \\
6-10=2,11-15=3 \\
16-20=4,>20=5\end{array}$} & 1.00 & Count & 40 & 10 & 1 & 51 \\
\hline & & $\begin{array}{l}\% w \text { ithin } 1-5 \text { DAY } S=1 \\
6-10=2,11-15=3 \\
16-20=4,>20=5\end{array}$ & $78.4 \%$ & $19.6 \%$ & $2.0 \%$ & $100.0 \%$ \\
\hline & & $\%$ of Total & $40.0 \%$ & $10.0 \%$ & $1.0 \%$ & $51.0 \%$ \\
\hline & 2.00 & Count & 5 & 3 & 6 & 14 \\
\hline & & $\begin{array}{l}\% w \text { ithin } 1-5 \text { DAYS }=1 \\
6-10=2,11-15=3 \\
16-20=4,>20=5\end{array}$ & $35.7 \%$ & $21.4 \%$ & $42.9 \%$ & $100.0 \%$ \\
\hline & & $\%$ of Total & $5.0 \%$ & $3.0 \%$ & $6.0 \%$ & $14.0 \%$ \\
\hline & 3.00 & Count & 4 & 4 & & 8 \\
\hline & & $\begin{array}{l}\% w \text { ithin } 1-5 \text { DAYS }=1 \\
6-10=2,11-15=3 \\
16-20=4,>20=5\end{array}$ & $50.0 \%$ & $50.0 \%$ & & $100.0 \%$ \\
\hline & & $\%$ of Total & $4.0 \%$ & $4.0 \%$ & & $8.0 \%$ \\
\hline & 4.00 & Count & 3 & 2 & & 5 \\
\hline & & $\begin{array}{l}\% w \text { ithin } 1-5 \text { DAY } S=1 \\
6-10=2,11-15=3 \\
16-20=4,>20=5\end{array}$ & $60.0 \%$ & $40.0 \%$ & & $100.0 \%$ \\
\hline & & $\%$ of Total & $3.0 \%$ & $2.0 \%$ & & $5.0 \%$ \\
\hline & 5.00 & Count & 4 & 8 & 10 & 22 \\
\hline & & $\begin{array}{l}\% \text { w ithin } 1-5 \text { DAYS }=1 \\
6-10=2,11-15=3 \\
16-20=4,>20=5\end{array}$ & $18.2 \%$ & $36.4 \%$ & $45.5 \%$ & $100.0 \%$ \\
\hline & & $\%$ of Total & $4.0 \%$ & $8.0 \%$ & $10.0 \%$ & $22.0 \%$ \\
\hline \multirow[t]{3}{*}{ Total } & & Count & 56 & 27 & 17 & 100 \\
\hline & & $\begin{array}{l}\% w \text { ithin } 1-5 \text { DAY } S=1 \\
6-10=2,11-15=3 \\
16-20=4,>20=5\end{array}$ & $56.0 \%$ & $27.0 \%$ & $17.0 \%$ & $100.0 \%$ \\
\hline & & $\%$ of Total & $56.0 \%$ & $27.0 \%$ & $17.0 \%$ & $100.0 \%$ \\
\hline
\end{tabular}

Chi-Square Tests

\begin{tabular}{|c|c|c|c|}
\hline & Value & df & $\begin{array}{l}\text { As ymp. Sig. } \\
\text { (2-sided) }\end{array}$ \\
\hline Pearson Chi-Square & 40.063 & 8 & .000 \\
\hline Likelihood Ratio & 42.886 & 8 & .001 \\
\hline $\begin{array}{l}\text { Linear-by-Linear } \\
\text { As sociation }\end{array}$ & 23.075 & 1 & .001 \\
\hline $\mathrm{N}$ of Valid Cases & 100 & & \\
\hline
\end{tabular}

Sym metric Measures

\begin{tabular}{|l|r|r|}
\hline & Value & Approx. Sig. \\
\hline Pearson's R & .833 & .001 \\
Spearman Correlation & .830 & .001 \\
\hline
\end{tabular}

$* \mathbf{P}<\mathbf{0 . 0 5}=$ Significant

$* \mathbf{P}>\mathbf{0 . 0 5}=$ Not Significant

*Moderate Risk and High Risk Shows Highly and Greater Significant

*Moderate Risk and High Risk Shows Duration of Hospital Stay in Days Increases

* Pearson Chi-Square Test Shows Statistical Difference between Groups, P Value and Significance

The above data shows mechanism of injury playing a vital role in deciding the outcome of injury. More grievous the mechanism of injury more severe the injury which is reflected by increase in the hospital stay. 
An Observational Study of Outcome in Blunt Abdominal Trauma Managed Conservatively.

Table 16: Cross Tabulation of Data of Build and Nutrition by Complications

NONE=1,SEPSIS=2,M I=3, WOUND INFECTION AND ANASTOM SIS=4, INTESTINAL OBSTRUCTION=5,WOUND NFECT ION=6, PNEM ONIA=7, HYPERTENSIV E CRISIS=8, KETOACIDOSIS=9, PLEURAL EFFUSION=10,PULM ONARY $E M B O L I S M=11, P N E M$ ONIA AND DVT=12,ARDS=13, M .I WITH ARDS $=14$ * LOW RISK=1, MODERATE RISK=2, HIGH RISK $=3$ Crosstabulation

\begin{tabular}{|c|c|c|c|c|c|c|}
\hline & & & LOW RIS & $\begin{array}{l}\text { MODERA } \\
\mathrm{H} \text { RISK }=\end{array}$ & $\mathrm{IISK}=2$ & \\
\hline & & & 1.00 & 2.00 & 3.00 & Total \\
\hline $\mathrm{NONE}=1, \mathrm{SEPSIS}=2, \mathrm{M}$ & 1.00 & Count & 24 & 23 & 10 & 57 \\
\hline$=3$,WOUND & & $\%$ of Total & $24.0 \%$ & $23.0 \%$ & $10.0 \%$ & $57.0 \%$ \\
\hline $\begin{array}{l}\text { NFECTION AND } \\
\text { ANASTOMSIS }=4 \text {,INTES }\end{array}$ & 2.00 & Count & 1 & 6 & 2 & 9 \\
\hline TINAL & & $\%$ of Total & $1.0 \%$ & $6.0 \%$ & $2.0 \%$ & $9.0 \%$ \\
\hline OBSTRUCTION=5, WO & 3.00 & Count & 3 & & & 3 \\
\hline UND & & $\%$ of Total & $3.0 \%$ & & & $3.0 \%$ \\
\hline $\begin{array}{l}\text { INFECTION=6,PNEMO } \\
\text { NIA=7, HY PERTENSN E }\end{array}$ & 4.00 & $\begin{array}{l}\text { Count } \\
\% \text { of Total }\end{array}$ & $\begin{array}{r}1 \\
1.0 \%\end{array}$ & & & $\begin{array}{r}1 \\
1.0 \%\end{array}$ \\
\hline CRISIS=8, KETOACIDO & 5.00 & Count & 1 & 2 & 1 & 4 \\
\hline EFFUSION=10,PULMO & & $\%$ of Total & $1.0 \%$ & $2.0 \%$ & $1.0 \%$ & $4.0 \%$ \\
\hline NARY & 6.00 & Count & & 3 & 2 & 5 \\
\hline EMBOLISM=11,PNEMO & & $\%$ of Total & & $3.0 \%$ & $2.0 \%$ & $5.0 \%$ \\
\hline $\begin{array}{l}\text { NIA AND } \\
\text { DVT=12, ARDS }=13, \mathrm{M} . \mathrm{I}\end{array}$ & 7.00 & $\begin{array}{l}\text { Count } \\
\% \text { of Total }\end{array}$ & $\begin{array}{r}2 \\
2.0 \%\end{array}$ & $\begin{array}{r}2 \\
2.0 \%\end{array}$ & $\begin{array}{r}2 \\
2.0 \%\end{array}$ & $\begin{array}{r}6 \\
6.0 \%\end{array}$ \\
\hline WTH ARDS=14 & 8.00 & Count & 2 & 2 & & 4 \\
\hline & & $\%$ of Total & $2.0 \%$ & $2.0 \%$ & & $4.0 \%$ \\
\hline & 9.00 & Count & 3 & & & 3 \\
\hline & & $\%$ of Total & $3.0 \%$ & & & $3.0 \%$ \\
\hline & 10.00 & Count & & 1 & 1 & 2 \\
\hline & & $\%$ of Total & & $1.0 \%$ & $1.0 \%$ & $2.0 \%$ \\
\hline & 11.00 & Count & & 1 & & 1 \\
\hline & & $\%$ of Total & & $1.0 \%$ & & $1.0 \%$ \\
\hline & 12.00 & Count & 1 & & & 1 \\
\hline & & $\%$ of Total & $1.0 \%$ & & & $1.0 \%$ \\
\hline & 13.00 & Count & & 1 & 1 & 2 \\
\hline & & $\%$ of Total & & $1.0 \%$ & $1.0 \%$ & $2.0 \%$ \\
\hline & 14.00 & Count & 1 & & 1 & 2 \\
\hline & & $\%$ of Total & $1.0 \%$ & & $1.0 \%$ & $2.0 \%$ \\
\hline Total & & Count & 39 & 41 & 20 & 100 \\
\hline & & $\%$ of Total & $39.0 \%$ & $41.0 \%$ & $20.0 \%$ & $100.0 \%$ \\
\hline
\end{tabular}

Chi-Square Tests

\begin{tabular}{|l|r|r|r|}
\hline & Value & df & \multicolumn{1}{|c|}{$\begin{array}{c}\text { Asymp. Sig. } \\
\text { (2-sided) }\end{array}$} \\
\hline Pearson Chi-Square & 58.142 & 26 & .003 \\
Likelihood Ratio & 35.487 & 26 & .001 \\
Linear-by-Linear & 34.426 & 1 & .004 \\
As sociation & 100 & & \\
N of Valid Cases & & & \\
\hline
\end{tabular}

\section{Symmetric Measures}

\begin{tabular}{|l|r|r|}
\hline & Value & Approx. Sig. \\
\hline Pearson's R & .714 & .004 \\
Spearman Correlation & .702 & .004 \\
\hline
\end{tabular}

$* \mathbf{P}<0.05$ = Significant

$* \mathbf{P}>\mathbf{0 . 0 5}=$ Not Significant

*Moderate Risk And High Risk Shows Highly And Greater Significant* Pearson Chi-Square Test Shows Statistical Difference Between Groups, P Value And Significance

The above table shows that patients with good built and nutrition underwent less number of complications than compared to patients with poor built and nutrition 
An Observational Study of Outcome in Blunt Abdominal Trauma Managed Conservatively.

Table 17: Descriptive Statistics Mean and Standard Deviation

Descriptive Statistics

\begin{tabular}{|c|c|c|c|}
\hline & $\mathrm{N}$ & Mean & Std. Deviation \\
\hline AGE & 100 & 1.7900 & .7426 \\
\hline SEX & 100 & 1.1500 & .3589 \\
\hline MECHANISM OF INUURY & 100 & 1.6100 & .7640 \\
\hline COMORBIDITIES & 100 & 1.4500 & .7160 \\
\hline BUILT AND NUTRTION & 100 & 1.8100 & .7480 \\
\hline DISTRACT & 100 & 1.4600 & .7166 \\
\hline AGE IN RANGE & 100 & 1.8100 & .7480 \\
\hline $\begin{array}{l}\text { DURATION OF HOSPITAL } \\
\text { STAY }\end{array}$ & 100 & 2.3300 & 1.6395 \\
\hline $\begin{array}{l}\text { RISK PERCENTAGELOW } \\
\text { RISK }\end{array}$ & 100 & 2.5900 & .9331 \\
\hline $\begin{array}{l}\text { RISK PERCENTAGE } \\
\text { MODERA TE RISK }\end{array}$ & 100 & 1.7000 & 5774 \\
\hline $\begin{array}{l}\text { RISK PERCENTAGE } \\
\text { HIGH RISK }\end{array}$ & 100 & 1.2000 & .4495 \\
\hline DIAGNOSIS & 100 & 4.6300 & 2.9359 \\
\hline CONSERVATIVE MGMT & 100 & 1.1700 & .3775 \\
\hline $\begin{array}{l}\text { DELA YED LAPAROTOMY } \\
\text { MGMT }\end{array}$ & 100 & 1.8300 & .3775 \\
\hline COMPLICATIONS & 100 & 3.6100 & 3.9668 \\
\hline MORTA LITY & 100 & 1.0300 & .1714 \\
\hline ETIOLOGY & 100 & 1.6200 & .9617 \\
\hline Valid N (listwise) & 100 & & \\
\hline
\end{tabular}

Table 18: Descriptive Statistics Mean, Standard Deviation and Range

De scriptive Statistics

\begin{tabular}{|l|r|r|r|r|r|}
\hline & \multicolumn{1}{|c|}{$\mathrm{N}$} & \multicolumn{1}{c|}{ Mnimum } & Maximum & \multicolumn{1}{c|}{ Mean } & Std. Deviation \\
\hline AGE1 & 100 & 18.00 & 90.00 & 37.9200 & 16.6634 \\
Valid N (listw ise) & 100 & & & & \\
\hline
\end{tabular}

Table 19: Chi-Square Test for Conservative

\begin{tabular}{|l|l|l|l|l|l|}
\hline Conservative & Number of Cases & Percentage & Chi-Value & P-Value & Result \\
\hline Low Risk & 64 & 77.1 & 42.662 & 0.002 & Significant \\
\hline Moderate Risk & 19 & 22.9 & 44.582 & 0.001 & Significant \\
\hline High Risk & 0 & 0 & 0 & 0.190 & Not Significant \\
\hline Total & 83 & 100 & & & \\
\hline
\end{tabular}

\section{$P<0.05=$ Significant}

$P>0.05=$ Not Significant

Tabulated Value $=3.84$

* Moderate Risk Shows Highly Significant

* Chi-Square Test Shows Statistical Difference Between Groups, P Value And Significance

Table 20: Chi-Square Test for Delayed Laparotomy

\begin{tabular}{|l|l|l|l|l|l|}
\hline Delayed Laparotomy & Number Of Cases & Percentage & Chi-Value & P-Value & Result \\
\hline Low Risk & 0 & 0 & 0 & 0.114 & Not Significant \\
\hline Moderate Risk & 6 & 35.3 & 43.026 & 0.002 & Significant \\
\hline High Risk & 11 & 64.7 & 80.597 & 0.001 & Highly Significant \\
\hline Total & 17 & & & & \\
\hline
\end{tabular}

$P<0.05=$ Significant

$P>0.05=$ Not Significant

Tabulated Value $=3.84$

* High Risk in Delayed Laparotomy Shows Highly Significant

*Moderate Risk and High Risk Shows Significant and Highly Significant

* Chi-Square Test Shows Statistical Difference between Groups, P Value and Significance 
The above data shows that the score we have put forward validates as patients with low risk i.e $77.1 \%$ and patients with moderate risk $22.9 \%$ of the total 83 cases managed conservatively did not need any surgical intervention.

The second table shows that 17 out of the 100 cases who underwent delayed laparotomy out of which $64.7 \%$ were marked high risk and $35.3 \%$ were moderate risk were predicted to undergo surgical intervention which again validates our score.

\section{Discussion}

Injuries account for at least $10 \%$ of deaths worldwide, and low- and middle-income countries are disproportionately affected (WHO 2010). As these countries industrialize, the burden of injuries would continue to grow (Mock BWHO 2005, WHO DVIPD 2009). Many injury-related deaths can be avoided with established injury prevention strategies and improvements in trauma care, but this requires carefully orchestrated systems of prevention and care(Mock Lancet 2004, Mock WJS 2012). In India, the WHO estimates that $10 \%$ of deaths and $13 \%$ of disability-adjusted life years lost are due to injury (WHO 2009).

Blunt abdominal trauma, amongst injuries, causes significant morbidity and mortality. This study concentrates on the various aspects of blunt trauma.

Mechanisms of injury have major impact on the patient outcomes. The most common cause of blunt trauma abdomen reflected in our study was road traffic accident accounting to $63 \%$ of the cases. This was comparable to various other studies in India and worldwide. Mohapatra et al have attributed 62\% cases of blunt trauma to abdomen to RTA. Study by Curie et al has also reported 58.6\% cases due to RTAs.(6)(7)Fall from height formed the second most common cause $(21 \%)$ followed by assault $(9 \%)$ and hit or fall of heavy object $(7 \%)(8)$.

In our study most of the patients were in the third to fifth decade of life forming a total of $81 \%$. Only $19 \%$ were in the age group above 50 years. The mean age group in our study was 37 years. Many of the studies done showed similar results. A study by Richard curie showed maximum cases in the second to fifth decade of life $(59 \%)$ with mean age of 28 years.

Like most of the studies, our study also shows domination by males. Around $85 \%$ cases in our study were males. Tripathi et al have also reported $71 \%$ male patients in their study with a male to female ratio of 4.4:1(6)

Out of our 100 cases, $96 \%$ presented with abdominal tenderness with local or generalised guarding present in $62 \%$, coastal margin tenderness in $53 \%$, flank pain $29 \%$, pelvic tenderness $25 \%$, haematuria in $19 \%$, abdominal distension in $12 \%$ and guarding in only $12 \%$ of patients. Our study is comparable to Tripati et al which reported abdominal tenderness and guarding in $80 \%$ and $58 \%$ as two of the most common presentation of blunt abdominal trauma(6)(7)(8).

Hospital stay reflects the number of days required for treatment which in turn reflects the final outcome. The maximum hospital stay was 45 days as seen by us. It was seen mostly in the post operative cases with complications like anastomotic leak and also with co morbidities like diabetes mellitus and associated other injuries like long bone fractures. It was observed that patients managed conservatively had complications that arose from co-morbid illnesses like diabetes, ischemic heart disease and hypertension. In our study the duration of hospital stay ranged from 1 -45 days. Patient without co-morbid conditions had a minimum stay range from 1-5 days. These patients formed $42 \%$ cases, while patients with co-morbid conditions of moderate and high risk formed $8 \%$ and $1 \%$ respectively. Patients with stay ranging from 6-10 days had patients with no co-morbid conditions $9 \%$ with moderate risk of co-morbid condition 3\% and high risk of $2 \%$. Patients with stay of 11-15 days had patients with no co morbid condition as $3 \%$, moderate risk of $3 \%$ and high risk of $2 \%$. Patients having stay of 16-20 days had 3\% of cases with no co-morbid condition while moderate risk and high risk had $2 \%$ and $0 \%$ respectively. Only total of $22 \%$ of total cases had stay ranging from more than 20 days out of which high risk category formed $8 \%$. Our study indicates that patients with known co-morbid conditions like ischemic heart disease, diabetes and hypertension had increased hospital stay and these factors definitely had a significant impact on patient outcome.

Patients with distracting injuries like chest trauma, fracture of upper limb and lower limb when present with abdominal trauma increased the hospital stay and also affected the outcome. Such patients when labelled as high risk $13 \%$ showed significant change when compared to patients with no other injuries $67 \%$. Of these 67 cases, $92 \%$ had stay ranging from 1-5 days while those cases of high risk where distracting injuries were present $40.9 \%$. The 13 cases of high risk category had hospital stay more than 20 days. This was vital in proving that distracting injuries directly affected the outcome of patients who were conservatively managed.

Age plays an important role when it comes to the final outcome of injury to patients. It is evident that in absence of any co-morbid or distracting injuries younger age group 18-30 fair better than older age group of $31-50$ and above 50 constituting $43.1 \%, 35.3 \%$ and $21 \%$ when it comes to minimum hospital stay. On the contrary, age group of $31-50$ constituted $45.5 \%$ of the cases who had maximum duration of stay in hospital more 
than 20 days. This reflected that as age increases, patients tend to develop more complications in either conservative or operative group. This also affects the hospital stay and the response to treatment.

Our study observed that the knowledge of mechanism of injury played an important part as far as predicting the injury pattern and its outcome. The study showed that more grievous the mechanism, longer the duration of treatment. High risk injury formed $45.5 \%$ out of the total 22 cases who had prolonged treatment compared to just $18.2 \%$ in low risk category. While in trivial or low risk, mechanism of injury formed $78.4 \%$ of the total 51 cases which had minimum stay for treatment. A meagre $2 \%$ of patients with high risk had treatment duration of less than 5 days.

In our study, out of 100 cases, 43 percent had complications.Most of the patients managed conservatively did not have any complications which formed $61.4 \%$ of total cases managed conservatively. Most common complication was sepsis and pneumonia constituting $9 \%$ and $6 \%$ respectively. Out of the 43 patients who had complications $25.6 \%$ occurred post operatively forming $64.7 \%$ of total number of operative cases. While $74.4 \%$ of cases belonged to the ones managed conservatively. Reasons for occurrence of complications in conservatively managed patients were poor build and nutrition, older age group, pallor on presentation and presence of distracting injuries. This reflected that complications occured less in patients managed conservatively as compared to operative patients. Complications in the patients managed conservatively occurred due to causes other than the trauma like myocardial infarction, ketoacidosis which got aggravated due to trauma and played an important role in outcome.

\section{Conclusion}

An accurate method for quantitatively summarizing injury severity has many potential applications. The ability to predict outcome from trauma (ie, mortality) is perhaps the most fundamental use of injury severity scoring, a use that arises from the patient's and the family's desires to know the prognosis. More recently, physicians suggested that injury severity scoring can provide objective information for end-of-life decisionmaking and resource allocation. Trauma mortality prediction in individual patients by any scoring system is limited and is in general no better than good clinical judgment. Therefore, decisions for individual patients should never be based solely on a statistically derived injury severity score. However, scoring systems can serve to estimate quantitatively the level of acuity of injured patients that are applied to adjustments in hospital outcome assessments.

\section{References}

[1] Fitzgerald M, Dewan Y, O’Reilly G. India and the management of road crashes: Towards a national trauma system. Indian J Surg. 2006 ;

[2] Joshipura MK, Mock C, Goosen J PM. strengthening trauma systems round the world. Essent Trauma Care. 2004;3:841-2.

[3] MK J. Total Trauma Care:International Perspective Hospital Today. Hosp Today. 1996;11:43-4.

[4] Dove DB ,Stahl WM DGC. a five year review of death following trauma. J trauma 20. :760.

[5] London PS. Abdominal injuries -- surgical aspects: a review. J R Soc Med.1979 Nov;72(11):842-5.

[6] Tripathi MD , Srivastava RD NA. Blunt abdominal traumawith special reference to early detection of visceral injuries. Indian J surg1991. 53AD;5:179-84.

[7] Richard A.Curie ALW. Blunt abdominal trauma. Am J surgsurg. 1964;107:321-7.

[8] Mohapatra S, Prahad S RK. Options in the management of solid visceral injuries from blunt abdominal trauma. indian J Surg. $2003 ; 65(3): 263-8$ 